\title{
Neutron capture cross sections from Surrogate measurements
}

\author{
Jutta E. Escher ${ }^{\mathrm{a}}$, Frank S. Dietrich, and Nicholas D. Scielzo \\ Lawrence Livermore National Laboratory, Livermore, CA, USA
}

\begin{abstract}
The prospects for determining cross sections for compound-nuclear neutron-capture reactions from Surrogate measurements are investigated. Calculations as well as experimental results are presented that test the Weisskopf-Ewing approximation, which is employed in most analyses of Surrogate data. It is concluded that, in general, one has to go beyond this approximation in order to obtain $(\mathrm{n}, \gamma)$ cross sections of sufficient accuracy for most astrophysical and nuclear-energy applications.
\end{abstract}

\section{Introduction}

Cross sections for compound-nuclear $(\mathrm{n}, \gamma)$ reactions are needed for a variety of applications, including astrophysics and nuclear energy. Modeling astrophysical processes that produce the heavy isotopes beyond iron, simulating nuclear reactor operations, exploring alternative fuel cycles for energy generation, and studying transmutation options for radioactive waste, requires cross sections for neutroninduced reactions on isotopes from different regions of the nuclear chart. As many short-lived species cannot be made into targets for direct cross-section measurements, one has to rely on calculations or explore indirect approaches.

The desired accuracies for the cross sections of interest, often in the range of $10 \%$ or less, can be much smaller than the theoretical uncertainties that exist when the model parameters are insufficiently constrained by data. For instance, standard evaluations for the $(\mathrm{n}, \gamma)$ reaction on the s-process branch point nucleus ${ }^{95} \mathrm{Zr}\left(t_{1 / 2}=64 \mathrm{~d}\right)$ vary from each other roughly by a factor of four ${ }^{1}$. Exploiting regional systematics, whenever cross sections or relevant structural data (level densities, $\gamma$-ray strength functions, etc.) for nearby nuclei are known, can provide valuable constraints for the calculations.

In this contribution we explore the prospects for determining or constraining $(\mathrm{n}, \gamma)$ cross sections through Surrogate measurements. The Surrogate nuclear reaction technique combines experiment with theory to obtain cross sections for compound-nuclear $(\mathrm{CN})$ reactions, $a+A \rightarrow B^{*} \rightarrow$ $c+C$, involving targets $(A)$ that are difficult or impossible to obtain [1-3]. In the Hauser-Feshbach formalism, the cross section for this "desired" reaction takes the form:

$$
\sigma_{\alpha \chi}\left(E_{a}\right)=\sum_{J, \pi} \sigma_{\alpha}^{C N}\left(E_{e x}, J, \pi\right) G_{\chi}^{C N}\left(E_{e x}, J, \pi\right)
$$

\footnotetext{
e-mail: escher1@1lnl.gov

1 Based on a comparison of ENDF/B-VII, JEFF-3.1, JENDL3.3, and ROSFOND evaluations provided through the database of the National Nuclear Data Center (NDDC) in November 2009
}

with $\alpha$ and $\chi$ denoting the relevant entrance and exit channels, $a+A$ and $c+C$, respectively. The excitation energy $E_{e x}$ of the compound nucleus, $B^{*}$, is related to the centerof-mass energy $E_{a}$ in the entrance channel via the energy needed for separating $a$ from $B: E_{a}=E_{e x}-S_{a}(B)$. In many cases the formation cross section $\sigma\left(a+A \rightarrow B^{*}\right)=$ $\sigma_{\alpha}^{C N}\left(E_{\alpha}\right)=\sum_{J \pi} \sigma_{\alpha}^{C N}\left(E_{e x}, J, \pi\right)$ can be calculated to a reasonable accuracy by using optical potentials, while the theoretical decay probabilities $G_{\chi}^{C N}\left(E_{e x}, J, \pi\right)$ for the different decay channels $\chi$ are often quite uncertain. The objective of the Surrogate method is to determine or constrain these decay probabilities experimentally.

In the Surrogate approach, the compound nucleus $B^{*}$ is produced by means of an alternative ("Surrogate"), direct reaction, $d+D \rightarrow b+B^{*}$, and the desired decay channel $\chi\left(B^{*} \rightarrow c+C\right)$ is observed in coincidence with the outgoing particle $b$ (see Fig. 1). The coincidence measurement provides

$$
P_{\delta \chi}\left(E_{e x}\right)=\sum_{J, \pi} F_{\delta}^{C N}\left(E_{e x}, J, \pi\right) G_{\chi}^{C N}\left(E_{e x}, J, \pi\right),
$$

which is the probability that the $\mathrm{CN}$ was formed in the Surrogate reaction with spin-parity distribution $F_{\delta}^{C N}\left(E_{e x}, J, \pi\right)$ and subsequently decayed into the channel $\chi$. The distribution $F_{\delta}^{C N}\left(E_{e x}, J, \pi\right)$, which may be very different from the $\mathrm{CN}$ spin-parity populations following the absorption of the projectile $a$ in the desired reaction, has to be determined theoretically, so that the branching ratios $G_{\chi}^{C N}\left(E_{e x}, J, \pi\right)$ can be extracted from the measurements. In practice, the decay of the $\mathrm{CN}$ is modeled and the $G_{\chi}^{C N}\left(E_{e x}, J, \pi\right)$ are obtained by adjusting parameters in the model to reproduce the measured probabilities $P_{\delta \chi}\left(E_{e x}\right)[4,5]$. Subsequently, the sought-after cross section can be obtained by combining the calculated cross sections $\sigma_{\alpha}^{C N}\left(E_{e x}, J, \pi\right)$ for the formation of $B^{*}$ (from $a+A$ ) with the extracted decay probabilities $G_{\chi}^{C N}\left(E_{e x}, J, \pi\right)$ for this state.

In Section 2 we will consider an application of the method to the the ${ }^{155} \mathrm{Gd}(\mathrm{n}, \gamma)$ reaction. The relevant compound nucleus is ${ }^{156} \mathrm{Gd}$, which was, in this case discussed, produced via inelastic proton scattering on the stable ${ }^{156} \mathrm{Gd}$

This is an Open Access article distributed under the terms of the Creative Commons Attribution-Noncommercial License, which permits unrestricted use, distribution, and reproduction in any noncommercial medium, provided the original work is properly cited. 

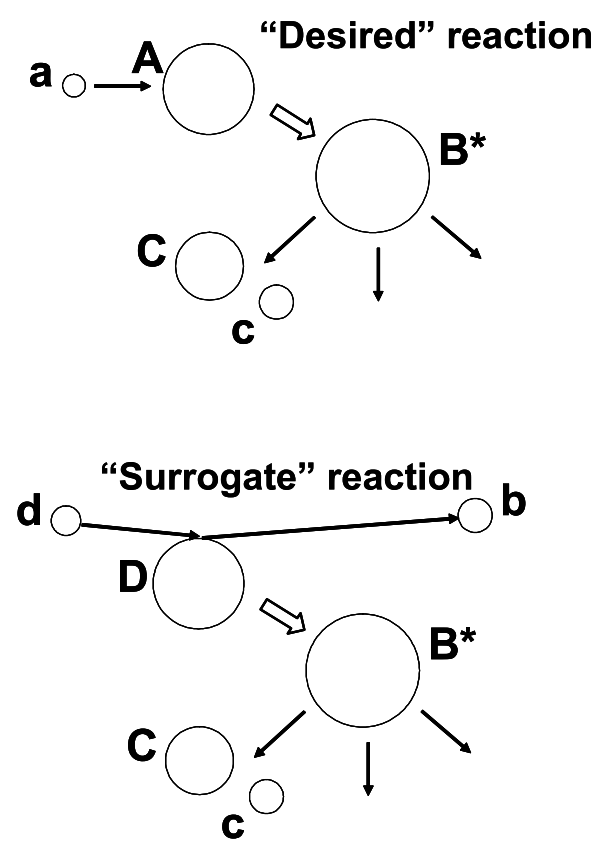

Fig. 1. Schematic representation of the "desired" (top) and "Surrogate" (bottom) reaction mechanisms. The basic idea of the Surrogate approach is to replace the first step of the desired reaction, $a+A$, by an alternative (Surrogate) reaction, $d+D \rightarrow b+B^{*}$, that populates the same compound nucleus. The subsequent decay of the compound nucleus into the relevant channel, $c+C$, can then be measured and used to extract the desired cross section.

ground state. The $\gamma$ exit channel can be identified by measuring $\gamma$-rays characteristic of electromagnetic transitions between levels of ${ }^{154} \mathrm{Gd}$ (such as transitions between members of the rotational band built on the ground state).

The Surrogate method was originally developed in the 1970s [1,2] and has primarily been used to obtain (n,f) cross sections. Almost all analyses of Surrogate data to date have made use of the Weisskopf-Ewing approximation (or the related Surrogate Ratio approach [3]). In the Weisskopf-Ewing limit of the Hauser-Feshbach formalism, the branching ratios become independent of spin and parity, $G_{\chi}(E, J, \pi) \rightarrow \mathcal{G}(E)$, which greatly simplifies the description of both the desired reaction,

$$
\sigma_{\alpha \chi}(E) \rightarrow \sigma_{\alpha \chi}^{W E}(E)=\sigma_{\alpha}^{C N}(E) \mathcal{G}_{\chi}^{C N}(E),
$$

and the Surrogate measurement,

$$
P_{\delta \chi}(E) \rightarrow P_{\delta \chi}^{W E}(E)=\mathcal{G}_{\chi}^{C N}(E) .
$$

This approximation has been found to work reasonably well for determining $(\mathrm{n}, \mathrm{f})$ cross sections, as long as one considers neutron energies above about 1 or $2 \mathrm{MeV}$. The extracted cross sections are typically in reasonable agreement with direct measurements $(\approx 10-15 \%)$, when the latter are available, or with results from other Surrogate experiments. The approach has provided valuable new data for cases where direct measurements were either not possible or covered only a limited energy range. Examples include $(\mathrm{n}, \mathrm{f})$ cross sections for ${ }^{237} \mathrm{U}$ [6] and ${ }^{233} \mathrm{~Pa}$ [7].

\section{Obtaining (n, $\gamma)$ cross sections from Surrogate measurements}

For low energies $\left(E_{n}<1-2 \mathrm{MeV}\right)$ the conditions for the Weisskopf-Ewing approximation are not expected to be well satisfied [3] and it becomes necessary to account for the "spin-parity mismatch," that is differences between the spin-parity distributions of the compound nuclei produced in the desired and Surrogate reactions, respectively. Introducing such corrections in the analysis of Surrogate fission data has been shown to improve the agreement with direct measurements $[4,5]$. Since the low-energy regime is important for many $(\mathrm{n}, \gamma)$ cross sections needed for astrophysical and nuclear-energy applications, one would expect that the Weisskopf-Ewing approximation does not provide cross sections of sufficient accuracy.

\subsection{Gamma decay probabilities and the Weisskopf-Ewing approximation}

For the Zirconium region, the validity of the WeisskopfEwing approximation was studied theoretically by Forssén et al. [8]. The dependence of the $\gamma$-decay probabilities $G_{\gamma}(E, J, \pi)$ on spin and parity was investigated and (n, $\left.\gamma\right)$ cross sections were extracted from a Weisskopf-Ewing analysis of simulated Surrogate data. To obtain the branching ratios, a Hauser-Feshbach calculation for ${ }^{91} \mathrm{Zr}(\mathrm{n}, \gamma)$ was carried out, with parameters adjusted to reproduce known structural data (s-wave resonance spacing, average radiative widths), as well as directly-measured cross sections. The fitting procedure defined a model for the decay of the $\mathrm{CN}^{92} \mathrm{Zr}$ and made it possible to extract theoretical branching ratios for the $\gamma$ channel. In Fig. 2 we show the resulting $\gamma$-decay probabilities, $G_{\gamma}(E, J, \pi=-)$, for angular momenta $J=0,3,6,9,12 \hbar$ and excitation energies $E_{e x}=8.64-12.5$ $\mathrm{MeV}$, which correspond to neutron energies, $E_{n}=E_{e x}-S_{n}$, in the range of $0-4 \mathrm{MeV}$ (in Ref. [8] $J$ values $0-4 \hbar$ and both positive and negative parities were considered).

Fig. 2 illustrates one of the main insights gained by the study of Ref. [8], the fact that the branching ratios $G_{\gamma}(E, J, \pi)$ depend very sensitively on angular momentum and parity of the decaying nucleus. In the energy regime considered, the decay of the ${ }^{92} \mathrm{Zr} \mathrm{CN}$ proceeds primarily by $\gamma$ or neutron emission, with negligible contributions from other channels. Due to the low level density in the neighboring nucleus ${ }^{91} \mathrm{Zr}$, very few neutron decay channels are available; the opening of each new channel corresponds to a discontinuity in one or more $\gamma$-branching ratios. This circumstance, and the fact that the neutron decay is dominated by low partial waves (mainly s and $\mathrm{p}$ wave), leads to $\gamma$-decay probabilities that are very sensitive to the $J \pi$ population of the decaying compound state. It is clear that the Weisskopf-Ewing approximation is not valid in this region.

The situation is expected to improve as one moves away from closed shells. For example, while ${ }^{91} \mathrm{Zr}$ has only one level below $1 \mathrm{MeV}$ (the ground state), the well-deformed rare-earth nucleus ${ }^{155} \mathrm{Gd}$ has over 60 , and the actinide nucleus ${ }^{235} \mathrm{U}$ has approximately 90 . Consequently, the decay probabilities for ${ }^{156} \mathrm{Gd}$ and ${ }^{236} \mathrm{U}$ depend more smoothly on 

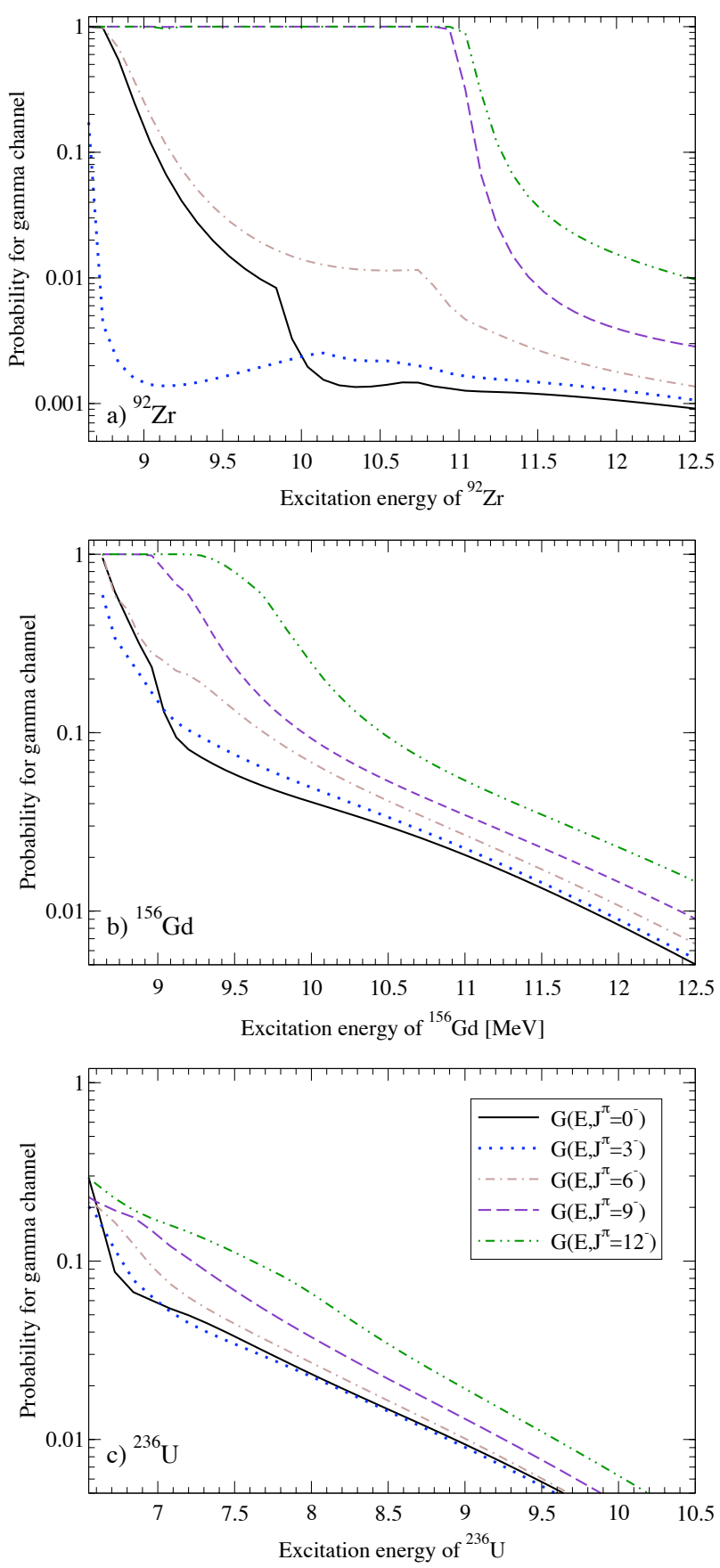

Fig. 2. Calculated $\gamma$-decay probabilities $G_{\gamma}^{C N}(E, J, \pi)$, for ${ }^{92} \mathrm{Zr}$, ${ }^{156} \mathrm{Gd}$, and ${ }^{236} \mathrm{U}$. Shown is the probability that the compound nucleus, when produced with a specific $J \pi$ combination, decays via the $\gamma$ channel. The excitation energies shown correspond to incident-neutron energies of $0-4 \mathrm{MeV}$. Only negative-parity decay probabilities are given. Note that the scale for the $y$ axis of panel (a) differs from that for panels (b) and (c).

energy and exhibit significantly less sensitivity to the $J \pi$ values of the compound nucleus. This is shown in panels (b) and (c) of Figure. 2, where the same branching ratios are plotted for the $\gamma$-decays of ${ }^{156} \mathrm{Gd}$ and ${ }^{236} \mathrm{U}$, respectively. Overall, the curves for the heavier nuclei show a much smoother behavior than those for the Zirconium case. Moreover, for large enough energies, the shape of the probabilities becomes almost independent of spin (for the range of spins considered, $J=0-12 \hbar$ ); for ${ }^{156} \mathrm{Gd}$ this occurs at $E_{e x} \approx 10.0-10.5 \mathrm{MeV}$, i.e. $E_{n} \approx 1.5-2.0 \mathrm{MeV}$, while for ${ }^{236} \mathrm{U}$ the $G_{\gamma}(E, J, \pi=-)$ have similar shapes for excitation energies above about 7.0-7.5 MeV, i.e. neutron energies higher than $E_{n} \approx 0.5-1.0 \mathrm{MeV}$. For ${ }^{156} \mathrm{Gd}$, the curves for the highest $J$ value, $J=12 \hbar$, are larger in magnitude than those for $J=0,3 \hbar$ by a factor of about 2.5-3 in this higher-energy regime; for ${ }^{236} \mathrm{U}$, the difference is somewhat smaller, around 2.0-2.5. The results shown in Figure 2 indicate that the Weisskopf-Ewing approximation is less likely to be valid in lower-mass nuclei, and particularly near closed shells, where the level densities are low.

\subsection{Cross sections from Weisskopf-Ewing analyses}

Whether it is reasonable to employ the Weisskopf-Ewing approximation for a particular reaction depends not only on the energy regime considered, but also on the range of angular momenta populated in both the desired and Surrogate reactions. It is possible that one reaction, for example the desired reaction, populates a narrow range of spins, while the other involves a wider range of angularmomentum values.

The effects of the spin-parity mismatch can be further explored by using schematic spin-parity distributions, $F_{\delta}^{C N}$ $(E, J, \pi)$, to simulate Surrogate coincidence data via Eq. 2. The calculated $P_{\delta \gamma}^{s i m}(E)=\sum_{J, \pi} F_{\delta}^{C N}(E, J, \pi) G_{\gamma}^{C N}(E, J, \pi)$ can be used in a Weisskopf-Ewing 'analysis' to yield the desired $(\mathrm{n}, \gamma)$ cross section, $\sigma_{n, \gamma}^{W E, s i m}(E)=\sigma_{n+\text { target }}(E) P_{\delta \gamma}^{\text {sim }}(E)$, where $\sigma_{n+\text { target }}(E)$ denotes the compound-nucleus formation cross section. The range of cross sections, $\sigma_{n, \gamma}^{W E, s i m}(E)$, obtained by varying the simulated spin distributions within reasonable limits provides a measure of the uncertainty in the extracted cross section due to the use of the Weisskopf-Ewing approximation. For the Zirconium region such sensitivity analysis was carried out by Forssén et al. [8]. An order-of-magnitude difference between the known reference cross section for ${ }^{91} \operatorname{Zr}(\mathrm{n}, \gamma)$ and that extracted from the simulation was found, indicating that using the Weisskopf-Ewing approximation for the this region of the nuclear chart is not appropriate.

For the rare-earth and actinide cases, the discrepancies are expected to be smaller. Recent studies [9] show that this is indeed the case. Results for the ${ }^{155} \mathrm{Gd}(\mathrm{n}, \gamma)$ example are shown in Fig. 3. Plotted are the reference cross section (solid curve), obtained by fitting a Hauser-Feshbach calculation to direct measurements (filled circles with $\mathrm{x}$ and $\mathrm{y}$ error bars), and three cross sections extracted from simulated Surrogate data (dotted, dash-dotted, and dashed curves). The associated spin distributions are shown in Fig. 4. Also shown in Fig. 3 are results from an actual Surrogate experiment, carried out by the STARS/ LIBERACE collaboration at the 88-inch cyclotron at Lawrence Berkeley Laboratory: The diamond-shaped symbols with y error bars indicate the cross section obtained from a Weisskopf-Ewing analysis of a ${ }^{156} \mathrm{Gd}\left(p, p^{\prime}\right)$ measurement with $22-\mathrm{MeV}$ protons. 
Details of the experiment and its analysis can be found in Refs. [10,11].

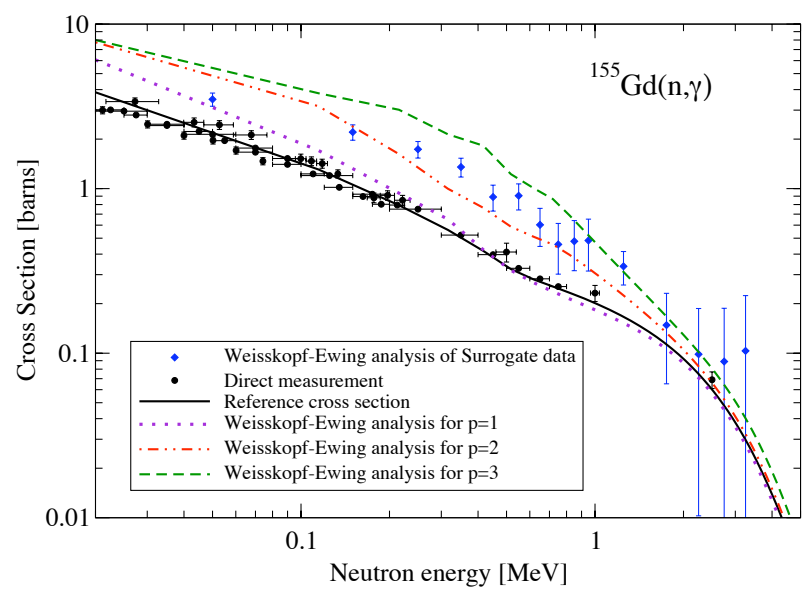

Fig. 3. Cross sections for ${ }^{155} \mathrm{Gd}(\mathrm{n}, \gamma)$. The cross section obtained from a Weisskopf-Ewing analysis of a Surrogate ${ }^{156} \mathrm{Gd}\left(p, p^{\prime}\right)$ experiment [11] (filled diamonds with y error bars) is compared to direct measurements (filled circles with $\mathrm{x}$ and $\mathrm{y}$ error bars) and a Hauser-Feshbach calculation (solid curve) for which the parameters were adjusted to reproduce the available data. Cross sections obtained from a Weisskopf-Ewing analysis of simulated surrogate measurements with spin-parity distributions $p=1,2,3$ (see Fig. 4) are shown as well.

For neutron energies below about $1.5 \mathrm{MeV}$, the cross sections extracted from the Weisskopf-Ewing analysis of the simulated Surrogate data are consistently too high, up to a factor of four. The discrepancies between the directlymeasured cross sections and those extracted from the Weisskopf-Ewing analysis of the Surrogate data can be understood with the help of the $\gamma$-decay probabilities $G_{\gamma}^{C N}(E, J, \pi)$ shown in Fig. 2b: If the Surrogate reaction populates the relevant compound nucleus, ${ }^{156} \mathrm{Gd}$ here, with a spin-parity distribution that contains larger angular-momentum values than the population relevant to the neutron-induced reaction, then the measured (or calculated) decay probability $P_{\delta \gamma}(E)$ of Eq. 2 contains larger contributions from those $G_{\gamma}^{C N}\left(E_{e x}, J, \pi\right)$ associated with large $J$ values than the cross section expression for the desired $(n, \gamma)$ reaction, Eq. 1, does. Consequently, the cross section extracted by using the Weisskopf-Ewing assumption and approximating $\mathcal{G}_{\gamma}^{C N}(E) \approx P_{\delta \gamma}(E)$, gives too large a result.

The largest deviations from the expected results occur for distribution $p=3$. This is the distribution that has the smallest overlap with the $J \pi$ population of the compound nucleus in the neutron-induced reaction. The spinparity distributions relevant to neutron-induced reactions for ${ }^{155} \mathrm{Gd}$ are not shown here; calculations illustrate [9] that the spin distributions for the desired reaction shift from angular-momentum values $J=1-3 \hbar$ at $0.1 \mathrm{MeV}$ to somewhat higher values with increasing energy, but for the energy range considered there is little to no contribution for angular momenta above 5-6ћ. Distributions that peak at low spins, such as distribution $p=1$, yield much closer
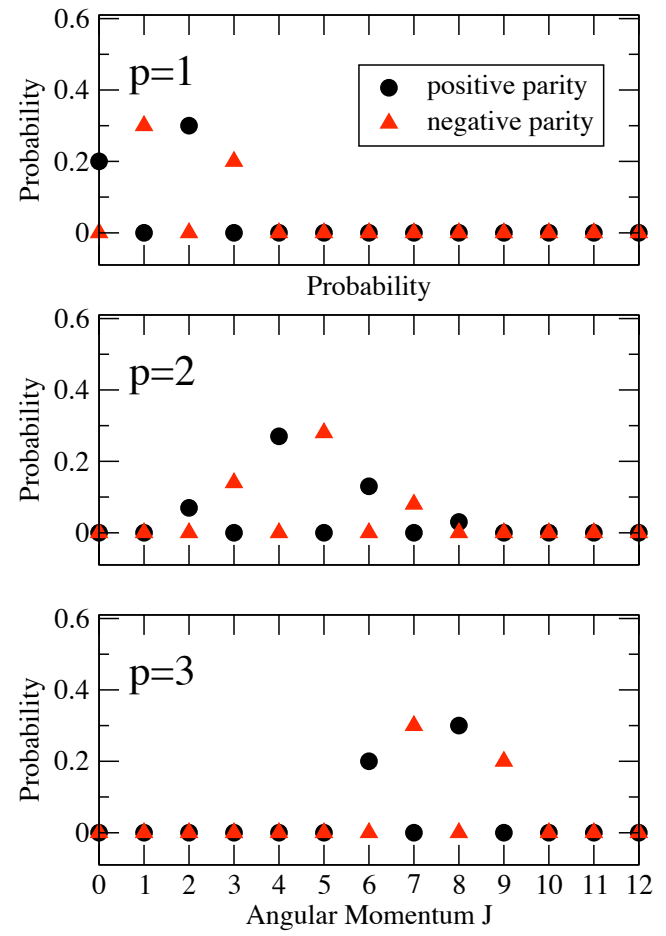

Fig. 4. Spin-parity distributions of the compound nucleus ${ }^{156} \mathrm{Gd}$. Three schematic spin-parity distributions, $p=1,2$, and 3 , were selected to simulate the compound nucleus prior to decay via $\gamma$ and neutron emission.

agreement with the reference cross section (for $E_{n}>0.4$ $\mathrm{MeV}$, the ${ }^{155} \mathrm{Gd}(\mathrm{n}, \gamma)$ results are within $\sim 25 \%$ of the expected result). If an experiment can be identified and carried out such that the reaction mechanism and experimental conditions (projectile energy, angle of detection of the outgoing direct-reaction particle, etc.) create $J \pi$ distributions similar to the one produced in the desired reaction, one can expect the cross sections extracted from a Weisskopf-Ewing analysis of the data to be in reasonable agreement with the true (n, $\gamma$ ) cross section. Presently, the compound-nuclear spin-parity distributions are not known for Surrogate reactions. Efforts are underway to develop methods for calculating these distributions and to test the theoretical predictions. This will make it possible to select a reaction mechanism and conditions that approximately reproduce the $J \pi$ distribution of the desired reaction and/or to correct for the mismatch.

Fig. 3 also shows the results obtained from a Weisskopf-Ewing analysis of the Surrogate data of Ref. [11]. The extracted cross section falls, for the most part, between the calculated curves. It is somewhat larger than the curve corresponding to distribution $p=2$, but smaller than the curve for $p=3$. This indicates that the $\left(p, p^{\prime}\right)$ reaction on ${ }^{156} \mathrm{Gd}$ produced a spin-parity distribution which contained $J$-values above $5-6 \hbar$. The cross section extracted from the Surrogate measurement is a factor of 2-3 larger than the reference cross section. Clearly, it is important to correct for the spin-parity mismatch if one wants to improve on this result. Efforts to do so are underway. 
The cross sections obtained from the simulations $p=$ $1,2,3$ are seen to converge for energies larger than about $1.5 \mathrm{MeV}$, i.e. the dependence on the spin-parity distribution decreases and the Weisskopf-Ewing assumption becomes a better approximation. In this energy region, the experimental results seem to be in rough agreement with the reference result (or possibly slightly too high), but the statistical uncertainties from the measurement become too large to draw more detailed conclusions.

\section{Summary and conclusions}

Motivated by the renewed interest in the Surrogate nuclear reactions approach, we have examined the prospects for determining $(\mathrm{n}, \gamma)$ cross sections for deformed rare-earth and actinide nuclei from Surrogate measurements. In particular, we investigated the validity of the Weisskopf-Ewing approximation, which is commonly employed when extracting $(\mathrm{n}, \mathrm{f})$ cross sections from Surrogate experiments. The Weisskopf-Ewing approach, which neglects the fact that the spin-parity population of the compound nucleus produced in the Surrogate reaction is different from that of the compound nucleus occurring in the desired reaction, was tested with calculations that simulated observables for typical Surrogate experiments. The approach used here is similar to the method employed in our earlier study of $(n, f)$ reactions [3] and complements and extends the investigation of $(n, \gamma)$ reactions for near-spherical nuclei in the mass 90-100 region [8]. The validity of the Surrogate Ratio Approach, which makes use of the Weisskopf-Ewing approximation, can be investigated analogously. This issue is studied elsewhere $[9,11]$.

Overall, we found that the probability for a compound nucleus to decay via $\gamma$ emission depends sensitively on the spin-parity population of the nucleus prior to decay. The dependence of the $\gamma$-branching ratios on the $J \pi$ distribution is greater than that found previously for fission. Calculations for representative Zirconium, Gadolinium, and Uranium nuclei showed a strong dependence of the $\gamma$ branching ratios on the spins populated in the compound nucleus, in particular for the lightest system considered here, the ${ }^{92} \mathrm{Zr}$ nucleus, which has a closed proton subshell $(\mathrm{Z}=40)$ and a nearly-closed neutron shell $(\mathrm{N}=52 \approx 50)$. A comparison with the results for Gadolinium and Uranium confirms the notion that the higher level densities present in the deformed rare-earth and actinide regions do indeed reduce the sensitivity of the $\gamma$-decay probabilities to compoundnuclear spin-parity distributions and nuclear-structure effects.

For Gadolinium, we also demonstrated that the (n, $\gamma$ ) cross sections obtained from a Weisskopf-Ewing analysis of Surrogate data can differ significantly from the expected 'true' cross section. The uncertainty seen in the cross sections extracted from simulated Surrogate measurements is clearly greater than that found previously for $(n, f)$ cross sections. It illustrates the limitations of this approximation when considering applications of the method to mass regions and/or types of reactions for which the method has not been tested yet.
We complemented our theoretical sensitivity studies with results from a recent Surrogate experiment. A Weisskopf-Ewing analysis of the ${ }^{156} \mathrm{Gd}\left(p, p^{\prime} \gamma\right)$ coincidence data measured by Scielzo et al. $[10,11]$ yielded a ${ }^{155} \mathrm{Gd}(\mathrm{n}, \gamma)$ cross section that differs up to a factor of three from the directly-measured cross section. These results are in agreement with our theoretical predictions and further underscore the need to account for the spin-parity mismatch between the Surrogate and desired reactions.

Measurements that test the validity of the Surrogate method are important and valuable. Applications of the method to $(\mathrm{n}, \mathrm{f})$ reactions have been tested in numerous experiments over the years. For $(\mathrm{n}, \gamma)$ reactions, only a few experiments exist [11-16]. Still fewer have been designed to properly test the method. In order to provide useful information on the validity and limitations of the method, Surrogate benchmark experiments need to yield cross section results that can be compared to direct measurements: The energy ranges covered by the direct and Surrogate measurements must have a sizable overlap, and the error bars associated with the two data sets have to be small enough to allow for a meaningful distinction between agreement and disagreement. Further, sufficient nuclear structure and reaction data should be available for the region to allow for calculations supporting the interpretation of the Surrogate measurement. It is also important to test the Weisskopf-Ewing approximation independently from the Ratio approach, as in the latter effects might cancel that have to be understood for a proper application of the Surrogate approach across a range of nuclei.

To move beyond the approximate analysis methods currently employed, a comprehensive theoretical treatment of the Surrogate approach is required. This involves a description of direct reactions that populate highly-excited, unbound states, and the damping of these doorway states into more complicated configurations that lead to a compound nucleus. The possibility that the intermediate system produced in a given Surrogate reaction does not lead to the compound nucleus of interest, but decays via nonequilibrium particle emission prior to reaching the compound stage, has to be considered. The probability for this process needs to be calculated, along with its dependence and influence on angular momentum, parity, and energy of the decaying nuclear system [17]. Developing a reliable theoretical description of the formation of a compound nucleus following a direct reaction will be crucially important for improving the accuracy and reliability of the Surrogate method and for extending its applicability beyond $(\mathrm{n}, \mathrm{f})$ reactions on actinide targets to other reaction types and mass regions.

While the strong spin-parity dependence of the observables used to tag the exit channel makes extracting $(\mathrm{n}, \gamma)$ cross sections from Surrogate measurements very challenging, it also provides valuable information. In particular, simultaneously measuring the yields of several $\gamma$-ray transitions of a decaying compound nucleus can provide signatures for the spin-parity distribution of the compound nucleus prior to decay. Relative $\gamma$-ray yields for the decay of even-even gadolinium nuclei have recently been measured $[10,11]$ and methods are being developed to use this 
information in order to improve the (n, $\gamma)$ cross sections determined from Surrogate experiments.

\section{Acknowledgments}

This work was performed under the auspices of the U.S. Department of Energy by Lawrence Livermore National Laboratory under contract DE-AC52-07NA27344.

\section{References}

1. J.D. Cramer, H.C. Britt, Nucl. Sci. and Eng. 41, 177 (1970)

2. H.C. Britt, J.B. Wilhelmy, Nucl. Sci. and Eng. 72, 222 (1979)

3. J.E. Escher, F.S. Dietrich, Phys. Rev. C 74, 054601 (2006)

4. W. Younes, H.C. Britt, Phys. Rev. C 67, 024610 (2003)

5. W. Younes, H.C. Britt, Phys. Rev. C 68, 034610 (2003)

6. J. Burke, L. Bernstein, J. Escher et al., Phys. Rev. C 73, 054604 (2006)

7. M. Petit, M. Aiche, G. Barreau et al., Nucl. Phys. A 735, 347 (2004)

8. C. Forssén, F. Dietrich, J. Escher, R. Hoffman, K. Kelley, Phys. Rev. C 75, 055807 (2007)

9. J.E. Escher, F.S. Dietrich (2009), submitted

10. N.D. Scielzo et al., AIP Conf. Proc. 1005, 109 (2008)

11. N.D. Scielzo et al. (2009), submitted

12. W. Peters et al. (2009), these proceedings

13. J.M. Allmond, L.A. Bernstein, C.W. Beausang, L. Phair, D.L. Bleuel, J.T. Burke, J.E. Escher, K.E. Evans, B.L. Goldblum, R. Hatarik et al., Phys. Rev. C 79, 054610 (2009)

14. B.L. Goldblum, S.G. Prussin, U. Agvaanluvsan, L.A. Bernstein, D.L. Bleuel, W. Younes, M. Guttormsen, Phys. Rev. C 78, 064606 (2008)

15. R. Hatarik et al., AIP Conf. Proc. 1005, 105 (2008)

16. S. Boyer, D. Dassie, J. Wilson, M. Aiche, G. Barreau, S. Czajkowski, C. Grosjean, A. Guiral, B. Haas, B. Osmanov et al., Nucl. Phys. A 775, 175 (2006)

17. F.S. Dietrich, AIP Conference Proceedings 1005, 125 (2008) 